\author{
() Т.П. Бардымова*, М.В. Мистяков, Г.П. Ягельская
}

Иркутская государственная медицинская академия последипломного образования - филиал Российской медицинской академии непрерывного профессионального образования, Иркутск

ОБОСНОВАНИЕ. В большинстве стран наблюдается стремительный рост популяции больных сахарным диабетом 2 типа (СД2). Костные изменения у женщин, больных СД2, в состоянии постменопаузы связаны с повышением минеральной плотности костной ткани (МПК). Продолжается изучение метаболических процессов в костной ткани при коморбидной патологии в разных этнических группах.

ЦЕЛЬ. Изучить концентрацию маркеров костного ремоделирования и показатели МПК у женщин бурятской популяции, больных СД2, в постменопаузальном периоде.

МЕтоды. Обследовано 39 женщин, больных СД2, постменопаузального периода (22 - русской популяции и 17 бурятской популяции). Группу сравнения составили 42 женщины в состоянии постменопаузы (21 - русской популяции и 21 - бурятской популяции). Исследование МПК в поясничном отделе позвоночника ( $\left.\mathrm{L}_{1}-\mathrm{L}_{\mathrm{IV}}\right)$, шейке бедра (Neck), в проксимальном отделе бедренной кости (Total hip) проводили с помощью двухэнергетической рентгеновской абсорбциометрии. Оценивали показатели остеокальцина (ОK), N-терминального пропептида проколлагена 1-го типа (P1NP), C-концевых телопептидов коллагена I типа ( $\beta$-Cross laps), 25(OH) витамина D и ионизированного кальция.

РЕзУЛЬтАТЫ. Представленное исследование выявило одновременное повышение показателей остеосинтеза: OK $(p=0,048)$ и P1NP ( $p=0,016)$, а также маркера костной резорбции $\beta$-Cross laps $(p=0,020)$, сопровождавшееся отсутствием изменений МПК у женщин бурятской популяции, больных СД2, в постменопаузальном периоде относительно женщин русской популяции с СД2 в состоянии постменопаузы. При СД2 у пациенток бурятской популяции в периоде постменопаузы установлено снижение показателей остеосинтеза (OK, p=0,021; P1NP, p=0,029) с повышением MПK $\mathrm{L}_{\mathrm{I}}-\mathrm{L}_{\mathrm{IV}}(\mathrm{p}=0,024)$ и MПК Total hip $(\mathrm{p}=0,039)$ относительно группы сравнения женщин бурятской популяции.

ЗАКЛЮЧЕНИЕ. Состояние костной ткани у женщин бурятской популяции, больных СД2, в постменопаузальном периоде характеризуется активацией процессов костного ремоделирования.

КЛЮЧЕВЫЕ СЛОВА: сахарный диабет 2 типа; костное ремоделирование; этнические особенности

\title{
CONDITION OF BONE TISSUE IN POSTMENOPAUSAL WOMEN WITH TYPE 2 DIABETES MELLITUS OF BURYAT POPULATION
}

(C) Tatiana P. Bardymova, Maksim V. Mistiakov, Galina P. Yagelskaya

Irkutsk State Medical Academy of Postgraduate Education - Branch Campus of the Russian Medical Academy of Continuing Professional Education, Irkutsk, Russia

BACKGRAUND: In most countries, there is a rapid increase in the population of patients with type 2 Diabetes Mellitus (DM). Bone changes in postmenopausal women with type $2 \mathrm{DM}$ are associated with increased bone mineral density (BMD). The study of metabolic processes in bone tissue in comorbid pathology in different ethnic groups is continuing.

AIMS: To study the concentration of markers of bone remodeling and indicators of BMD in postmenopausal women with type 2 DM of the Buryat population.

MATERIALS AND METHODS: Thirty-nine postmenopausal women with type 2 DM (22 - Russian population and 17 - Buryat population) were examined. The comparison group consisted of 42 postmenopausal women ( 21 - Russian population and 21 - Buryat population). The study of BMD in the lumbar spine (L1-L4), the femoral neck (Neck), and the proximal femur (Total hip) was performed using dual-energy X-ray absorptiometry. Parameters of osteocalcin (OC), type $1 \mathrm{~N}$-terminal procollagen propeptide (P1NP), C-terminal telopeptides of type I collagen ( $\beta$-Cross laps), $25(\mathrm{OH})$ vitamin $D$ and ionized calcium were evaluated.

RESULTS: The presented study revealed a simultaneous increase in osteosynthesis: $O C(p=0.048)$ and P1NP ( $p=0.016)$ and in the bone resorption marker $\beta$-Cross laps $(p=0.020)$ accompanied by the absence of changes in BMD in women with type 2 DM in the postmenopausal period of the Buryat population relative to women with type 2 DM in the postmenopausal state 
of the Russian population. A decrease in osteosynthesis parameters (OC, $p=0.021 ; P 1 N P, p=0.029)$ with an increase in $B M D$ L1-L4 $(p=0.024)$ and BMD Total hip $(p=0.039)$ in postmenopausal women with type 2 DM of the Buryat population was found relative to the women of the Buryat population in comparison group.

CONCLUSIONS: The state of bone tissue in postmenopausal women with type 2 DM of the Buryat population is characterized by the activation of bone remodeling processes.

KEYWORDS: diabetes mellitus, type 2; bone remodeling; ethnicity

\section{ОБОСНОВАНИЕ}

Сахарный диабет 2 типа (СД2) характеризуется развитием долгосрочных осложнений, к которым поражение костной ткани традиционно не относилось. Ряд исследований показал, что СД2 можно отнести к факторам риска переломов костей независимо от индекса массы тела (ИМТ) и повышенной частоты падений $[1,2]$. СД2 чаще встречается у женщин, и пик заболеваемости приходится на возраст старше 40 лет [3]. Как известно, с возрастом наблюдается более высокий риск остеопоротических переломов, а при диабете у женщин изменения костной ткани наслаиваются на состояние постменопаузы, с наступлением которой костные потери ускоряются [4]. Проведенные ранее исследования показали, что при СД2 отмечаются высокие показатели минеральной плотности костной ткани (МПК), нарушаются процессы костеобразования и костной резорбции [5-8]. В последние годы шире исследуется связь маркеров костного ремоделирования с показателями МПК и риском переломов при СД. Имеется недостаточно сведений о состоянии костного метаболизма у женщин монголоидного происхождения, данные носят противоречивый характер. До настоящего времени остаются малоизученными вопросы, связанные с изучением особенностей метаболических процессов костной ткани у женщин бурятской популяции, больных СД.

\section{ЦЕЛЬ}

Изучить маркеры костного ремоделирования и показатели МПК у женщин бурятской популяции, больных СД2, в постменопаузальном периоде.

\section{МЕТОДЫ}

\section{Дизайн исследования}

Проведено наблюдательное одноцентровое одномоментное выборочное контролируемое нерандомизированное исследование.

\section{Критерии соответствия}

Критерии включения в исследование: женский пол, принадлежность к русской или бурятской популяциям, возраст 50-65 лет, продолжительность менопаузы не менее одного года, диагноз СД2, отсутствие выраженных осложнений диабета.

К критериям исключения относились факторы риска развития вторичного остеопороза: системные ревматические заболевания (ревматоидный артрит, спондилоартрит и другие болезни соединительной ткани), заболевания органов желудочно-кишечного тракта с нарушением всасывания кальция (в том числе резекции желудка, син- дром мальабсорбции и др.), другие эндокринные заболевания (тиреотоксикоз, гиперкортицизм и др.), онкологические заболевания и болезни крови, хроническая болезнь почек 3-5-й стадий. Также не включались женщины на менопаузальной гормональной терапии, принимающие глюкокортикоидные препараты, иммунодепрессанты и препараты для лечения остеопороза (в том числе в анамнезе).

\section{Условия проведения}

Исследование проводилось (амбулаторно) на базе клинико-диагностического центра ИГМАПО - филиала ФГБОУ ДПО РМАНПО Минздрава России, г. Иркутск.

Продолжительность исследования

Исследование проведено в период с 2016 г. по 2018 г.

\section{Описание медицинского вмешательства}

После подписания информированного согласия у всех пациенток проводился анализ анамнестических данных, общеклинического обследования с занесением в анкеты, специально разработанные для исследования. Участницы исследования сдавали образцы крови утром натощак с 8 до 9 ч из кубитальной вены. Исследование МПК проводили методом двухэнергетической рентгеновской абсорбциометрии (DXA).

\section{Основной исход исследования}

Основными показателями, оцениваемыми в исследовании, были значения МПК и маркеры костного ремоделирования: остеокальцин (ОК) и N-терминальный пропептид проколлагена 1-го типа (P1NP) сыворотки крови, C-концевые телопептиды коллагена I типа ( $\beta$-Cross laps) плазмы крови у пациенток с СД2, в состоянии постменопаузы и групп сравнения женщин в периоде постменопаузы русской и бурятской популяций.

\section{Дополнительные исходы исследования}

Изучены уровень ионизированного кальция (iCa) плазмы крови и содержание $25(\mathrm{OH})$ витамина D в сыворотке крови у пациенток с СД2 в состоянии постменопаузы и групп сравнения женщин в периоде постменопаузы русской и бурятской популяций.

\section{Анализ в подгруппах}

Участниц исследования разделили на две когорты: когорту с СД2 и когорту сравнения.

В когорту с СД2 включили пациенток с верифицированным СД2. Верификация диагноза осуществлялась по данным медицинской документации на момент первичной диагностики (ВОЗ, 1999-2013).

В когорту сравнения включили пациенток без нарушений углеводного обмена. 
Дополнительно каждая когорта пациенток была разделена на две группы:

- русской популяции - в нее вошли пациентки, считавшие себя, своих родителей и их предков по этническому происхождению русскими;

- бурятской популяции - в нее вошли пациентки, считавшие себя, своих родителей и их предков по этническому происхождению бурятами.

\section{Методы регистрации исходов}

Методом DXA оценивали МПК в поясничном отделе позвоночника ( $\left.\mathrm{L}_{\mathrm{-}} \mathrm{L}_{\mathrm{IV}}\right)$, шейке бедра (Neck), в проксимальном отделе бедренной кости (Total hip). Исследования проводили на аппарате Prodigy, LUNAR (GE, USA).

Определение концентраций $\beta$-Cross laps и iCa плазмы крови, OK, P1NP и 25(OH) витамина D сыворотки крови проводили иммунохемилюминесцентным и ионоселективным методами. В работе использовали анализаторы Architect i2000, Cobas 601, Konelab PRIME 30 (Лаборатория «Инвитро»).

\section{Этическая экспертиза}

Проведение исследования одобрено Комитетом по этике научных исследований ИГМАПО - филиала ФГБОУ ДПО РМАНПО Минздрава России (протокол заседания № 1 от 28 января 2016 г.). Все обследованные женщины давали письменное информированное согласие на участие в исследовании.

\section{Статистический анализ}

Размер выборки предварительно не рассчитывался. Статистическая обработка данных проводилась с помощью прикладных программ Statistica 10.0 (StatSoft Inc., USA). Учитывая, что распределение большинства изученных признаков было отличным от нормального, применяли методы непараметрической статистики. Описательные статистические данные представлены в виде медианы и межквартильного интервала (Mе [Q1; Q3]). Вариационные ряды сравнивались по критерию МаннаУитни, основанному на сопоставлении средних значений в двух группах. Методом непараметрического корреляционного анализа (коэффициент корреляции Спирмена) оценивались ассоциации изучаемых показателей. Критический уровень значимости (р) для проверки статистиче- ских гипотез при сравнении статистических показателей принимался менее 0,05.

\section{РЕЗУЛЬТАТЫ}

Объекты (участники) исследования

В исследование включены 22 женщины с СД2 русской популяции, медиана возраста 57,5 [55; 62] лет, длительность заболевания от 6 до 11 лет, а также 17 пациенток с СД2 бурятской популяции, медиана возраста 59 [57; 61] лет, длительность заболевания от 7 до 12 лет. Группу сравнения составили 42 женщины без нарушений углеводного обмена, из них 21 женщина русской популяции, медиана возраста 56 [52; 60] лет, и 21 женщина бурятской популяции, медиана возраста 56 [52; 60] лет. Группы женщин с СД2 и группы сравнения по длительности постменопаузы были сопоставимы. Все женщины с СД2 были в состоянии удовлетворительной компенсации заболевания. Все пациентки с СД2 находились на стандартных схемах сахароснижающей терапии с учетом рациональных комбинаций.

\section{Основные результаты исследования}

Сравнительный анализ показал, что значения МПК Neck по результатам DXA у пациенток с СД2 русской популяции выше этих же показателей группы сравнения русских женщин ( $p=0,039)$. Кроме этого, у женщин с СД2 русской популяции отмечено снижение уровней P1NP $(p<0,0001)$, OK ( $p<0,0001)$ и $\beta$-Cross laps $(p=0,0002)$ относительно аналогичных данных группы сравнения русских женщин (табл. 1). У пациенток с СД2 русской популяции ИМТ был выше относительно русской группы сравнения $\left(30,9[27,4 ; 34,1] \mathrm{kг} / \mathrm{M}^{2}\right.$ и $26,4[25,6 ; 29,2] \mathrm{kг} / \mathrm{M}^{2}$ соответственно) $(p=0,025)$.

Отмечено, что у женщин, больных СД2, бурятской популяции повышались показатели МПК Total hip $(p=0,039)$ и МПK $\mathrm{L}_{1}-\mathrm{L}_{\mathrm{IV}}(\mathrm{p}=0,024)$ по сравнению с данными группы сравнения женщин-буряток. Кроме того, отмечена депрессия только остеосинтеза: снижение содержания ОК $(p=0,021)$ и P1NP $(p=0,029)$ у женщин-буряток с СД2 относительно соответствующей группы сравнения (табл. 2). У пациенток с СД2 бурятской популяции ИМТ также был выше относительно группы сравнения женщин-буряток

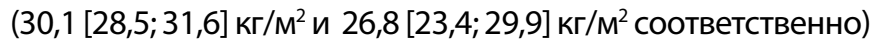
$(p=0,026)$.

Таблица 1. Сравнительная характеристика показателей двухэнергетической рентгеновской абсорбциометрии и маркеров костного ремоделирования у женщин русской популяции

\begin{tabular}{|c|c|c|c|c|c|c|}
\hline Показатель & $\begin{array}{c}\text { OK, } \\
\text { нг/мл }\end{array}$ & $\begin{array}{l}\text { P1NP, } \\
\text { нг/мл }\end{array}$ & $\begin{array}{c}\beta \text {-Cross laps, } \\
\text { нг/мл }\end{array}$ & 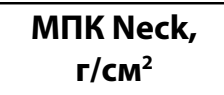 & 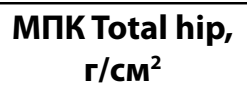 & 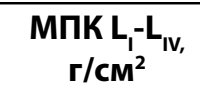 \\
\hline $\begin{array}{l}\text { Женщины } \\
\text { с СД2 } \\
(\mathrm{n}=22)\end{array}$ & $\begin{array}{c}14 \\
{[12 ; 17]^{*}}\end{array}$ & $\begin{array}{c}27,5 \\
{[22,6 ; 40,8]^{*}}\end{array}$ & $\begin{array}{c}0,254 \\
{[0,158 ; 0,409]^{*}}\end{array}$ & $\begin{array}{c}0,940 \\
{[0,886 ; 0,988]^{*}}\end{array}$ & $\begin{array}{c}1,024 \\
{[0,949 ; 1,072]}\end{array}$ & $\begin{array}{c}1,099 \\
{[1,025 ; 1,185]}\end{array}$ \\
\hline $\begin{array}{l}\text { Группа } \\
\text { сравнения } \\
(n=21)\end{array}$ & $\begin{array}{c}24 \\
{[21 ; 31]}\end{array}$ & $\begin{array}{c}49,7 \\
{[45,1 ; 61,9]}\end{array}$ & $\begin{array}{c}0,528 \\
{[0,411 ; 0,690]}\end{array}$ & $\begin{array}{c}0,864 \\
{[0,812 ; 0,935]}\end{array}$ & $\begin{array}{c}0,957 \\
{[0,885 ; 1,059]}\end{array}$ & $\begin{array}{c}1,058 \\
{[0,929 ; 1,125]}\end{array}$ \\
\hline
\end{tabular}

Примечания: * - статистически значимые отличия $(p<0,05)$.

OK - остеокальцин; P1NP - N-терминальный пропептид проколлагена 1-го типа; $\beta$-Cross laps - C-концевые телопептиды коллагена I типа; МпК минеральная плотность костной ткани; Neck - шейка бедра; Total hip - проксимальный отдел бедренной кости; $\mathrm{L}_{\mathrm{I}} \mathrm{L}_{\mathrm{IV}}-$ поясничный отдел позвоночника. 
Таблица 2. Сравнительная характеристика показателей двухэнергетической рентгеновской абсорбциометрии и маркеров костного ремоделирования у женщин бурятской популяции

\begin{tabular}{|c|c|c|c|c|c|c|}
\hline Показатель & $\begin{array}{l}\text { OK, } \\
\text { нг/мл }\end{array}$ & $\begin{array}{l}\text { P1NP, } \\
\text { Hг/Mл }\end{array}$ & $\begin{array}{c}\beta \text {-Cross laps, } \\
\text { нг/мл }\end{array}$ & $\begin{array}{l}\text { MПK Neck, } \\
\mathrm{r} / \mathrm{CM}^{2}\end{array}$ & $\begin{array}{l}\text { MПK Total hip, } \\
\mathbf{r} / \mathbf{c m}^{2}\end{array}$ & $\begin{array}{c}\text { MחK L } L_{1}-L_{1{ }^{\prime}} \\
r / \mathrm{Cm}^{2}\end{array}$ \\
\hline $\begin{array}{l}\text { Женщины } \\
\text { с СД2 } \\
(\mathrm{n}=17)\end{array}$ & $\begin{array}{c}16 \\
{[14 ; 22]^{*}}\end{array}$ & $\begin{array}{c}39,2 \\
{[32,8 ; 47,4]^{*}}\end{array}$ & $\begin{array}{c}0,450 \\
{[0,284 ; 0,560]}\end{array}$ & $\begin{array}{c}0,875 \\
{[0,759 ; 0,971]}\end{array}$ & $\begin{array}{c}1,002 \\
{[0,913 ; 1,075]^{*}}\end{array}$ & $\begin{array}{c}1,072 \\
{[1,015 ; 1,191]^{*}}\end{array}$ \\
\hline $\begin{array}{l}\text { Группа } \\
\text { сравнения } \\
(\mathrm{n}=21)\end{array}$ & $\begin{array}{c}23 \\
{[18 ; 28]}\end{array}$ & $\begin{array}{c}53,4 \\
{[42,5 ; 63,9]}\end{array}$ & $\begin{array}{c}0,555 \\
{[0,298 ; 0,664]}\end{array}$ & $\begin{array}{c}0,841 \\
{[0,763 ; 0,918]}\end{array}$ & $\begin{array}{c}0,897 \\
{[0,831 ; 0,966]}\end{array}$ & $\begin{array}{c}0,984 \\
{[0,893 ; 1,072]}\end{array}$ \\
\hline
\end{tabular}

Примечания: * - статистически значимые отличия $(p<0,05)$.

OK - остеокальцин; P1NP - N-терминальный пропептид проколлагена 1-го типа; $\beta$-Cross laps - C-концевые телопептиды коллагена I типа; МПК - минеральная плотность костной ткани; Neck - шейка бедра; Total hip - проксимальный отдел бедренной кости; $\mathrm{L}_{\mathrm{I}} \mathrm{L}_{\mathrm{IV}}-$ поясничный отдел позвоночника.
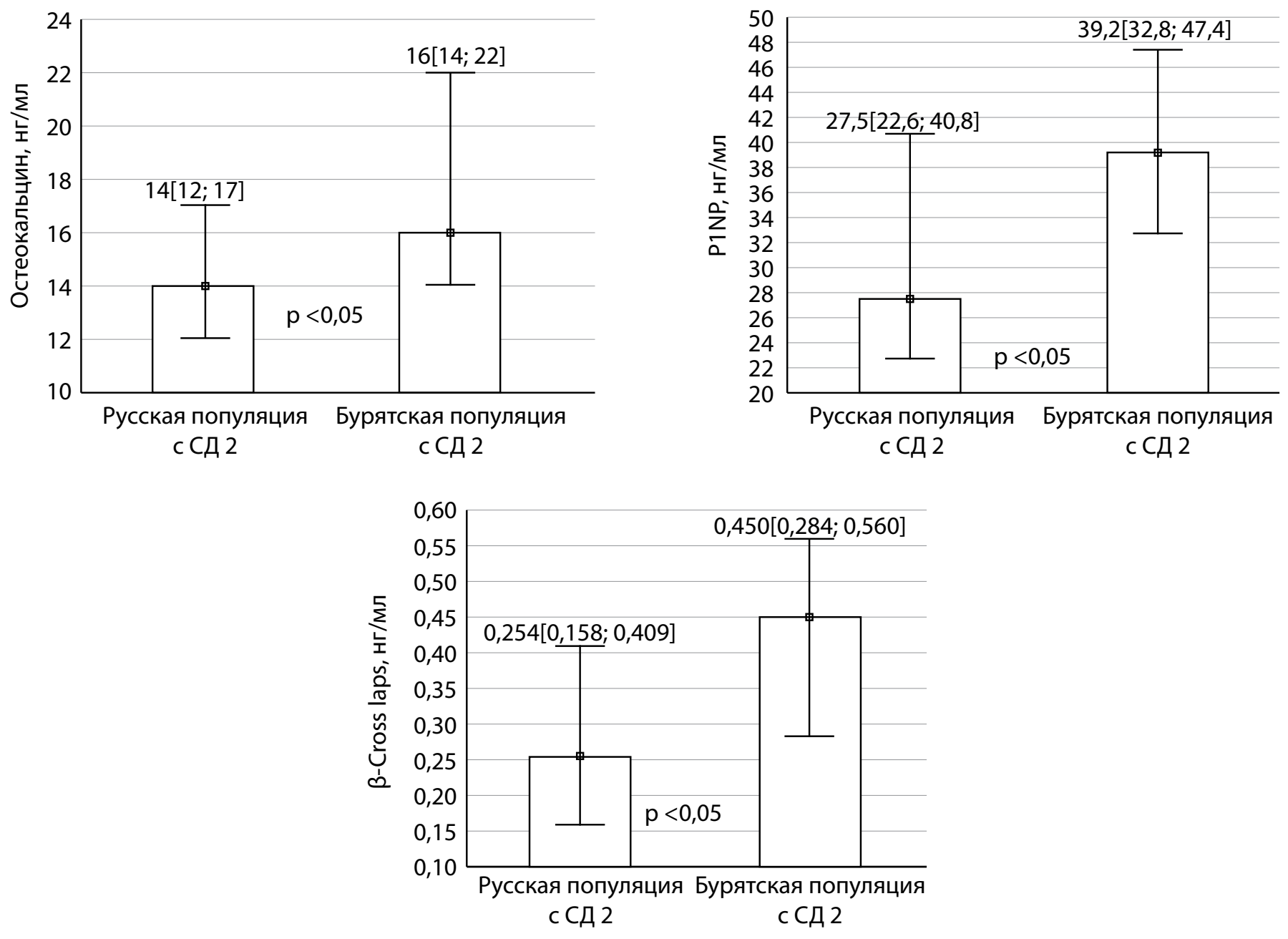

Рис. 1. Маркеры ремоделирования костной ткани у пациенток с сахарным диабетом 2 типа русской и бурятской популяций.

Установлены этнические особенности метаболизма костной ткани при СД2. Так, в группе женщин, больных СД2, бурятской популяции установлено ускорение ремоделирования костной ткани, характеризующееся повышением P1NP $(p=0,016)$, OK ( $p=0,048)$ и $\beta$-Cross laps $(p=0,02)$, относительно группы женщин с СД2 русской популяции (рис. 1). В то время как показатели МПК не различались между группами пациенток с СД изучаемых популяций ( $>>0,05)$.
Проведенный ранговый корреляционный анализ выявил значимую корреляцию концентрации 25(OH) витамина D с уровнями OK $(\rho=-0,69 ; p=0,002)$, P1NP $(\rho=-0,50 ; p=0,042)$ и $\beta$-Cross laps $(\rho=-0,55 ; p=0,023)$ у женщин, больных СД2, бурятской популяции (рис. 2). Кроме того, обнаружена взаимосвязь МПК Neck и P1NP в группе сравнения женщин-буряток $(\rho=0,57 ; p=0,017)$. 

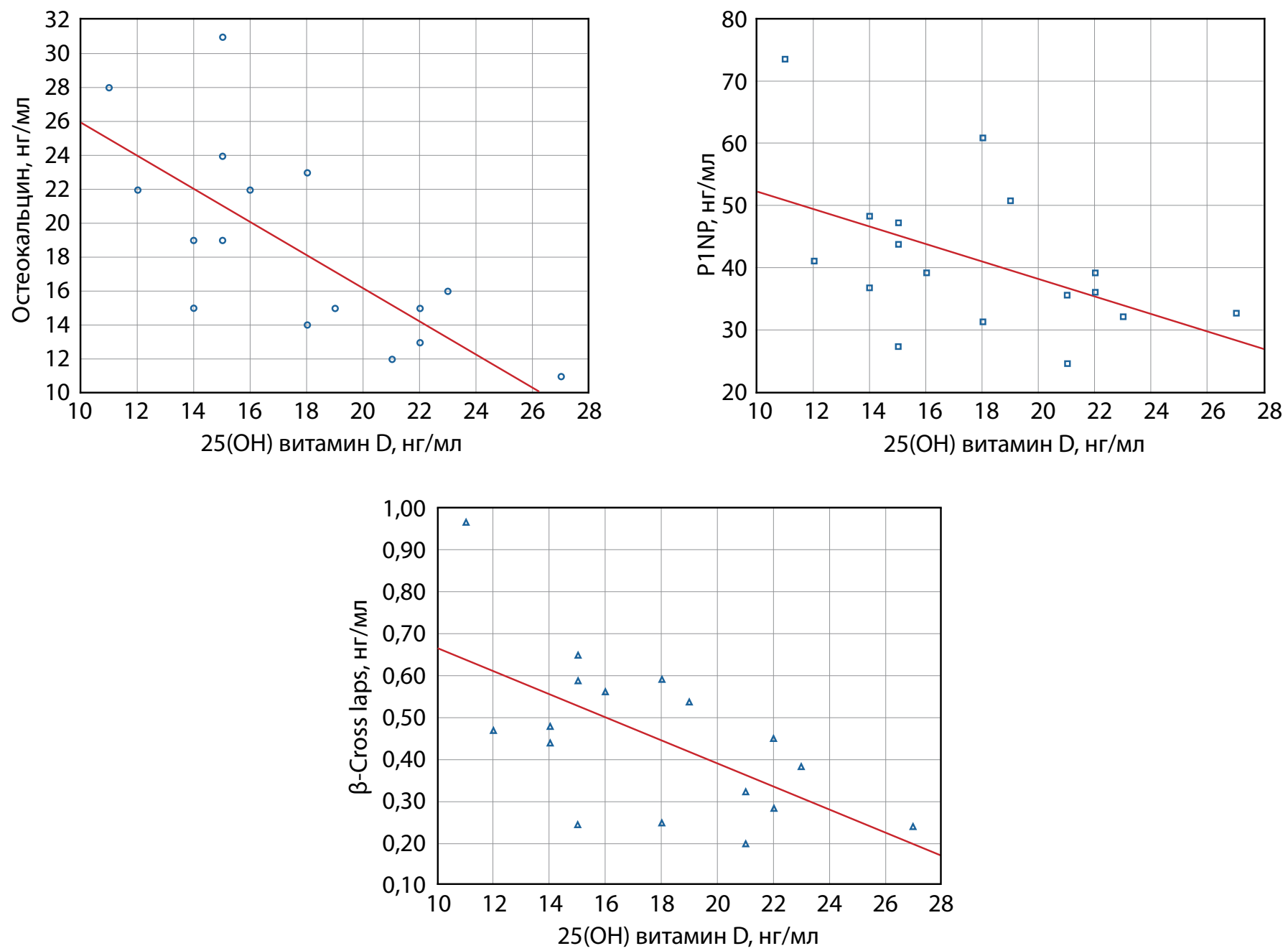

Рис. 2. Корреляционные связи между 25(OH) витамином D и маркерами ремоделирования костной ткани у пациенток с сахарным диабетом 2 типа бурятской популяциии

\section{Дополнительные результаты исследования}

У пациенток с СД2 русской популяции наблюдалось снижение уровня 25(OH) витамина D (20 [17; 27] нг/мл) относительно группы сравнения русских женщин (26 [21,5; 32] нг/мл) (p<0,05). У женщин, больных СД2, бурятской популяции показатель 25(ОН) витамина D был на уровне женщин-буряток группы сравнения (18 [15; 21] нг/мл и 18 [16; 22] соответственно) ( $>0,05)$. Одновременно не установлено различий в концентрации 25(OH) витамина D между группами женщин с СД2 русской и бурятской групп сравнения ( $>>0,05)$. Сравнительный анализ продемонстрировал особую стабильность іСа, концентрации которого между изучаемыми группами были сопоставимы и не отличались (p>0,05).

\section{Нежелательные явления}

Во время исследования нежелательных явлений не было отмечено.

\section{ОБСУЖДЕНИЕ}

\section{Резюме основного результата исследования}

Представленное исследование выявило одновременное повышение как показателей остеосинтеза, так и костной резорбции, сопровождавшееся отсутствием изменений МПК у женщин, больных СД2, в постменопа- узальном периоде бурятской популяции относительно женщин с СД2 в состоянии постменопаузы русской популяции. У пациенток с СД2 бурятской популяции в периоде постменопаузы установлено снижение показателей остеосинтеза (OK, P1NP) с повышением MПK L,- $\mathrm{L}_{\mathrm{IV}}$ и MПK Total hip относительно группы сравнения женщин бурятской популяции без СД2.

\section{Обсуждение основного результата исследования}

Повышение МПК у пациенток с СД2 согласуется с данными других исследователей, которые показали, что уровень МПК при СД2 превышает МПК лиц без диабета. Повышенную минерализацию костной ткани при СД длительно относили к протективным факторам, способствующим низкому риску низкотравматических переломов [9]. Все шире обсуждается вопрос увеличения риска развития переломов у пациентов с СД2, которые происходят при повышении МПК $[10,11]$. Результаты исследований позволяют предположить, что основной причиной костных нарушений при СД является не изменение МПК, а ухудшение качества кости и нарушения микроархитектоники костной ткани [11, 12].

В данном исследовании мы тестировали гипотезу об этнических особенностях метаболических процессов костной ткани у женщин, больных СД2, постменопаузального периода бурятской популяции. Нами 
установлено повышение МПК у пациенток с СД2 как русской, так и бурятской популяций относительно соответствующих этнических групп сравнения. Следует отметить, что локализация изменений МПК отличалась: у больных СД2 русской популяции - MПК Neck, а у пациенток с СД2 бурятской популяции - MПK $\mathrm{L}_{\mathrm{I}}-\mathrm{L}_{\mathrm{IV}}$ и МПК Total hip.

Как известно, на показатель МПК оказывает влияние скорость костного ремоделирования [13-15]. Состояние костного ремоделирования у женщин с СД2 в постменопаузальном периоде русской популяции характеризовалось замедлением как остеосинтеза (снижение P1NP, OK), так и резорбции костной ткани (снижение $\beta$-Cross laps) относительно русской группы сравнения. У пациенток с СД2 бурятской популяции установлено снижение только показателей P1NP, OK по сравнению с бурятской группой сравнения, что, видимо, предрасполагает к преобладанию костной резорбции над синтезом и будет способствовать риску переломов, в том числе низкотравматических

Результаты проведенных ранее работ показали, что при СД2 по сравнению с контрольной группой наблюдались низкие концентрации маркеров костного образования: OK и P1NP, а также маркера костной резорбции $\beta$-Cross laps $[5,6,8,16,17]$. Снижение $\beta$-Cross laps в группе русских пациенток с СД2 на фоне подавленного остеосинтеза свидетельствует о снижении скорости костного обмена, что согласуется с результатами метаанализа, проведенного Нуgum и соавт. (2017) [18].

По результатам проведенного исследования у женщин бурятской популяции, больных СД2, в периоде постменопаузы отмечено статистически значимое увеличение биохимических маркеров (P1NP, OK и $\beta$-Cross laps), отражающих интенсивность ремоделирования костной ткани и продемонстрировавших усиление как остеосинтеза, так и остеорезорбции по сравнению с пациентками с СД2 русской популяции. По отдельным исследованиям подобные данные могут способствовать изменениям биологии костной ткани. Установленный дисбаланс сывороточных маркеров костного обмена у буряток, больных диабетом, наблюдался на фоне стабильности МПК относительно группы женщин с СД2 в периоде постменопаузы русской популяции. Указывается, что на процессы костного ремоделирования при СД2 может оказывать влияние склеростин, который, высвобождаясь из остеоцитов, уменьшает дифференцировку и активность остеобластов, а также способствует снижению активности остеокластов. Снижение костного ремоделирования способствует накоплению престарелой костной массы, которая может накапливать микротрещины и имеет повышенный риск перелома независимо от МПК [19].

Полученные данные позволяют предположить, что выявленные особенности костного обмена у женщин бурятской популяции характеризуют диапазон компенсаторно-приспособительных реакций и регуляторных процессов, которые частично можно объяснить относительным влиянием эпигенетических факторов, связанных с разными анатомо-геометрическими характеристиками систем, в том числе структуры костной ткани, традиционного стереотипа питания, развитием адаптационных механизмов к болезни.
Общеизвестна роль 25-ОН витамина D, обеспечивающего абсорбцию кальция в кишечнике и оказывающего влияние на функционирование остеобластов и остеокластов [20]. Обнаруженное снижение уровня 25-OH витамина D у женщин, больных СД2, русской популяции относительно русской группы сравнения способствует прогрессированию остеопоретических процессов [21]. Концентрация 25-ОН витамина D у пациенток с СД2 бурятской популяции была на уровне бурятской группы сравнения, что подтверждается литературными данными об относительном снижении витамина D у азиатских народов [22]. Установленная обратная корреляционная связь концентрации 25-ОН витамина D с маркерами костного обмена у женщин, больных СД2, бурятской популяции может оказывать негативное влияние на процессы костного ремоделирования. Выявленная нами обратная связь согласуется с данными Jorde и соавт. (2019) [23].

\section{Ограничения исследования}

Одномоментный дизайн не позволяет судить о причинно-следственных взаимосвязях между признаками. Объем выборки требует осторожности при интерпретации полученных данных.

\section{ЗАКЛЮЧЕНИЕ}

Проведенная оценка состояния костной ткани позволяет установить этнические особенности обменных процессов, определяющих массу и метаболизм костной ткани, у женщин, больных СД2, в постменопаузальном периоде бурятской популяции. Так, в ходе исследования установлены низкие показатели OK, P1NP у женщин бурятской популяции, больных СД2, в постменопаузальном периоде относительно женщин группы сравнения бурятской популяции. В то же время у женщин с СД2 постменопаузального периода русской популяции также снижены OK, P1NP и дополнительно снижен показатель резорбции - $\beta$-Cross laps по сравнению с группой сравнения женщин русской популяции. У пациенток с СД2 выявлено повышение показателей МПК относительно соответствующих групп сравнения: шейки бедра - в русской популяции, проксимального отдела бедра и поясничного отдела позвоночника - в бурятской популяции. Сравнительный анализ маркеров костного ремоделирования при СД2 показал существенное повышение уровней OK, P1NP и $\beta$-Cross laps у женщин постменопаузального периода бурятской популяции относительно группы женщин в периоде постменопаузы русской популяции. В группе женщин бурятской популяции, больных СД2, в состоянии постменопаузы выявлена обратная корреляция между концентрацией 25-ОН витамина D и уровнями маркеров костного обмена. Как известно, каждый народ адаптирован к тем условиям, в которых он сформировался, и определение этнических категорий больных по отношению к метаболическим процессам позволит модифицировать существующие и разрабатываемые рекомендации с учетом этнических особенностей каждой группы пациентов. Предлагаемый подход позволит оптимизировать региональные программы профилактического характера, что будет способствовать своевременному выделению групп риска. 


\section{ДОПОЛНИТЕЛЬНАЯ ИНФОРМАЦИЯ}

Источник финансирования. Исследование проведено за счет внебюджетных средств организации.

Конфликт интересов. Авторы декларируют отсутствие явных и потенциальных конфликтов интересов, связанных с публикацией настоящей статьи.

Участие авторов. Бардымова Т.П. - концепция и дизайн исследования, анализ данных, написание текста; Мистяков М.В. - сбор клинического материала, статистическая обработка результатов, на- писание текста; Ягельская Г.П - анализ литературы, анализ данных. Все авторы внесли существенный вклад в проведение исследования и подготовку статьи, прочли и одобрили финальную версию перед публикацией.

Благодарности. Выражается благодарность всем пациентам, участвовавшим в данном исследовании, а также сотрудникам клинико-диагностического центра ИГМАПО - филиала ФГБОУ ДПО РМАНПО Минздрава России, Иркутского областного клинического консультативно-диагностического центра и Независимой лаборатории ИНВИТРО.

\section{СПИСОК ЛИТЕРАТУРЫ | REFERENCES}

1. Leslie WD, Rubin MR, Schwartz AV, et al. Type 2 diabetes and bone. J Bone Miner Res. 2012;27(11):2231-2237 doi: https://doi.org/10.1002/jbmr.1759

2. Ялочкина Т.О., Белая Ж.Е., Рожинская Л.Я., и др. Переломы костей при сахарном диабете 2 типа: распространенность и факторы риска // Сахарный диабет. - 2016. - Т. 19. - №5. - C. 359-365. [Yalochkina TO, Belaya JE, Rozhinskaya LY, et al. Bone fractures in patients with type 2 diabetes mellitus: prevalence and risk factors. Diabetes mellitus. 2016;19(5):359-365. (In Russ.)] doi: https://doi.org/10.14341/dm7796

3. Дедов И.И., Шестакова М.В., Викулова О.К., и др. Сахарный диабет в Российской Федерации: распространенность, заболеваемость, смертность, параметры углеводного обмена и структура сахароснижающей терапии по данным Федерального регистра сахарного диабета, статус 2017 г. // Сахарный диабет. - 2018. — T. 21. — №3. - C. 144-159. [Dedov II, Shestakova MV, Vikulova OK, et al. Diabetes mellitus in Russian Federation: prevalence, morbidity, mortality, parameters of glycaemic control and structure of hypoglycaemic therapy according to the Federal Diabetes Register, status 2017. Diabetes Mellitus. 2018;21(3):144-159. (In Russ.)] doi: https://doi.org/10.14341/DM9686

4. Boonen S, Body J, Boutsen Y, et al. Evidence-based guidelines for the treatment of postmenopausal osteoporosis: a consensus document of the Belgian Bone Club. Osteoporos Int. 2005;16(3):239-254. doi: 10.1007/s00198-004-1812-1

5. Starup-Linde J, Lykkeboe S, Gregersen S, et al. Differences in biochemical bone markers by diabetes type and the impact of glucose. Bone. 2016;83:149-155. doi: https://doi.org/10.1016/j.bone.2015.11.004

6. Jiajue $\mathrm{R}$, Jiang $\mathrm{Y}$, Wang $\mathrm{O}$, et al. Suppressed bone turnover was associated with increased osteoporotic fracture risks in non-obese postmenopausal Chinese women with type 2 diabetes mellitus. Osteoporos Int. 2014;25(8):1999-2005. doi: https://doi.org/10.1007/s00198-014-2714-5

7. Vestergaard P. Discrepancies in bone mineral density and fracture risk in patients with type 1 and type 2 diabetes a meta-analysis. Osteoporos Int. 2006;18(4):427-444. doi: https://doi.org/10.1007/s00198-006-0253-4

8. Бардымова Т.П., Мистяков М.В., Сеурко О.В. Маркеры костного метаболизма у женщин в постменопаузальном периоде с сахарным диабетом 2-го типа // Acta Biomedica Scientifica. 2017. — T. 2. — №1. — C. 16-18. [Bardymova TP, Mistyakov MV, Seurko OV. Metabolism. Acta Biomedica Scientifica. 2017;2(1):16-18. (In Russ.)]

9. Van Daele PL, Stolk RP, Burger H, et al. Bone density in non-insulin-dependent diabetes mellitus: the Rotterdam study. Ann Intern Med. 1995;22(6):409-414 doi: https://doi.org/10.7326/0003-4819-122-6-199503150-00002

10. Lecka-Czernik B. Diabetes, bone and glucose-lowering agents: basic biology. Diabetologia. 2017;60(7):1163-1169. doi: 10.1007/s00125-017-4269-4

11. Ferrari S, Abrahamsen B, Napoli $N$, et al. Diagnosis and management of bone fragility in diabetes: an emerging challenge. Osteoporos Int. 2018;29(12):2585-2596. doi: https://doi.org/10.1007/s00198-018-4650-2
12. Jackuliak P, Payer J. Corrigendum to "Osteoporosis, Fractures, and Diabetes". Int J Endocrinol. 2017;2017:2846080. doi: https://doi.org/10.1155/2017/2846080

13. Brandi ML. Microarchitecture, the key to bone quality. Rheumatology. 2009:48(Suppl 4):iv3-8. doi: https://doi.org/10.1093/rheumatology/kep273

14. Ulivieri FM, Silva BC, Sardanelli F, et al. Utility of the trabecular bone score (TBS) in secondary osteoporosis. Endocrine. 2014;47(2):435-448. doi: https://doi.org/10.1007/s12020-014-0280-4

15. Link TM, Majumdar S. Current diagnostic techniques in the evaluation of bone architecture. Curr Osteoporos Rep. 2004;2(2):47-52. doi: https://doi.org/10.1007/s11914-004-0003-5

16. Chen $\mathrm{H}, \mathrm{Li}$ X, Yue R, et al. The effects of diabetes mellitus and diabetic nephropathy on bone and mineral metabolism in T2DM patients. Diabetes Res Clin Pract. 2013;100(2):272-276. doi: https://doi.org/10.1016/j.diabres.2013.03.0077

17. Климонтов В.В., Фазуллина О.Н., Лыков А.П., и др. Взаимосвязь маркеров ремоделирования костной ткани с минеральной плотностью костей у женщин с сахарным диабетом 2 типа, находящихся в постменопаузе // Сахарный диабет. 2016. - T. 19. — №5. - C. 375-382. [Klimontov V, Fazullina ON, Lykov AP, et al. The relationships between bone turnover markers and bone mineral density in postmenopausal type 2 diabetic women. Diabetes mellitus. 2016;19(5):375-382. (In Russ.)] doi: https://doi.org/10.14341/DM8008

18. Hygum K, Starup-Linde J, Harslof T, et al. Mechanisms in endocrinology: Diabetes mellitus, a state of low bone turnover a systematic review and meta-analysis. Eur J Endocrinol. 2017;176(3):R137-157. doi: https://doi.org/10.1530/EJE-16-0652

19. Manolagas SC, Almeida M. Gone with the Wnts: betacatenin, T-cell factor, forkhead box $\mathrm{O}$, and oxidative stress in age dependent diseases of bone, lipid, and glucose metabolism. Mol Endocrinol. 2007;21(11):2605-2614. doi: https://doi.org/10.1210/me.2007-0259

20. Cranney A, Horsley T, O'Donnell S, et al. Effectiveness and safety of vitamin $D$ in relation to bone health. Rockville (MD): Agency for Healthcare Research and Quality (US); 2007. Available from: https://www.ncbi.n/m.nih.gov/books/NBK38410/

21. Пигарова Е.А., Рожинская Л.Я., Белая Ж.Е., и др. Клинические рекомендации Российской ассоциации эндокринологов по диагностике, лечению и профилактике дефицита витамина D у взрослых // Проблемы эндокринологии. — 2016. - T. 62. - №4. - C. 60-84. [Pigarova EA, RozhinskayaLYa, Belaya ZE, et al. Russian Association of endocrinologists recommendations for diagnosis, treatment and prevention of vitamin D deficiency in adults. Problems of Endocrinology. 2016;62(4):60-84. (In Russ.)] doi: https://doi.org/10.14341/probl201662460-84

22. Herrick KA, Storandt RJ, Afful J, et al. Vitamin D status in the United States, 2011-2014. Am J Clin Nutr. 2019;110(1):150-157. doi: https://doi.org/10.1093/ajcn/nqz037

23. Jorde R, Stunes AK, Kubiak J. Effects of vitamin D supplementation on bone turnover markers and other bone-related substances in subjects with vitamin D deficiency. Bone. 2019;124:7-13. doi: https://doi.org/10.1016/j.bone.2019.04.002 


\section{ИНФОРМАЦИЯ ОБ АВТОРАХ [AUTHORS INFO]}

*Бардымова Татьяна Прокопьевна, д.м.н., профессор [Tatiana P. Bardymova, MD, PhD, Professor]; адрес: Российская Федерация, 664049, Иркутск, мкр. Юбилейный, д. 100 [address: 100, mkr, Jubilejny, 664049 Irkutsk, Russian Federation]; ORCID: https://orcid.org/0000-0003-4241-2217; eLibrary SPIN: 6151-1430; e-mail: tpbardymova@mail.ru

Мистяков Максим Викторович [Maksim V. Mistiakov]; ORCID: https://orcid.org/0000-0002-4675-3396; eLibrary SPIN: 6430-3185; e-mail: mrdrx@mail.ru

Ягельская Галина Павловна [Galina P. Yagelskaya]; ORCID: https://orcid.org/0000-0001-8313-7974; e-mail:

gpyagelskaya@mail.ru

\section{ЦИТИРОВАТЬ:}

Бардымова Т.П., Мистяков М.В., Ягельская Г.П. Состояние костной ткани у женщин с сахарным диабетом 2 типа бурятской популяции в постменопаузальном периоде // Сахарный диабет. - 2019. — Т. 22. — №5. — С. $436-443$. doi: 10.14341/DM10310

\section{TO CITE THIS ARTICLE:}

Bardymova TP, Mistiakov MV, Yagelskaya GP. Condition of bone tissue in postmenopausal women with type 2 diabetes mellitus of buryat population. Diabetes Mellitus. 2019;22(5):436-443. doi: 10.14341/DM10310 